\title{
REMARQUES SUR LA THEORIE DES PROPRIÉTÉS MAGNETIQUES DES COUCHES MINCES ET DES GRAINS FINS
}

\author{
Par LouIS NÉEL.
}

\begin{abstract}
Summary. - The author shows, first, that in the case of powdered iron, the variation of the coercitive field against the grains'size, is correctly interpreted by supposing that the energy spent during a hysteresis cycle is only used for the irreversible production of the system of "walls" (produced twice in a cycle in the region where the resulting magnetisation nears zero). Agreement with theory is already less good for magnetite and becomes very bad for $\mathrm{Mn}-\mathrm{Bi}$ : for the latter, and generally for the substances with only one direction of easy magnetisation, the coercitive field is rather determined by the difficulties in forming the nuclei of inversion of the magnetisation. No satisfactory theory is now available for these phenomena of "nucleation": serious theoretical objections can be raised in fact against the theory proposed by Kittel.

In the second part, the author deals again with the theory of the coercitive field of thin continuous layers, and shows that the superficial energy of Bloch's "walls "has no constant value but increases as soon as the thickness of the wall is not negligible when compared with the thickness of the layer : let $0,1 \mu$ be the thickness of an iron layer, the "wall "energy is $7 \mathrm{erg} / \mathrm{cm}^{2}$, the normal value being $1,4 \mathrm{erg}-\mathrm{cm}^{2}$. It is perhaps an explanation of the increase of the coercitive field of thin layers when their thickness decreases.
\end{abstract}

1. Introduction. - Différents recoupements permettent d'établir que les couches magnétiques minces, c'est-à-dire pour fixer les idées, les couches d'épaisseur inférieure à $10^{-5} \mathrm{~cm}$, présentent selon les circonstances expérimentales soit une structure continue, soit une structure granulaire, et sont quelquefois constituées de grains complètement séparés les uns des autres, comme le montre notamment l'absence complète de conductibilité électrique. Malheureusement, très rares sont les cas où on connait exactement cette structure physique réelle, ce qui rend pratiquement impossible toute discussion précise des propriétés magnétiques.

D'autre part, en ce qui concerne la théorie des propriétés magnétiques, et plus spécialement la théorie du champ coercitif, on s'est généralement borné à assimiler les propriétés des couches continues très minces à celles des grains très fins alors qu'il existe des différences très profondes entre les deux. Il y a certainement un travail d'ensemble à reprendre à ce sujet mais qui dépasserait le cadre de cet exposé. Aussi, nous nous sommes bornés à reprendre ici quelques points très particuliers de la théorie des couches minces et des grains fins avec l'idée d'établir quelques bases de départ pour une théorie complète qu'il paraît prématuré d'établir maintenant.

Nous aborderons d'abord l'étude des grains fins, puis celle des couches minces.

2. La théorie de Kittel du champ coercitif des grains fins contenant plusieurs domaines élémentaires. - Lorsque les dimensions des grains d'une substance ferromagnétique sont inférieures à une certaine limite, qui est de l'ordre de grandeur de l'épaisseur des parois de Bloch, ces grains se comportent comme des domaines élémentaires uniques et le champ coercitif $H_{\mathrm{c}}$ prend une valeur généralement élevée. Ce champ $H_{\mathrm{c}}$ dépend de l'anisotropie des grains mais ne dépend pas en principe de leur volume. La théorie en a été complètement développée ailleurs [1].

Au contraire, à l'état massif, pur et non déformé, les ferromagnétiques possèdent un champ coercitif faible. Le simple bon sens suggère qu'en réduisant progressivement le diamètre $d$ des grains d'une poudre on doit observer une augmentation régulière du champ coercitif, depuis la valeur faible qui caractérise l'état massif jusqu'à la valeur limite élevée relative aux grains très fins. Effectivement, l'expérience montre [2] que $H_{\mathrm{c}}$ varie en gros comme $1 / d$.

Pour interpréter quantitativement ce phénomène, Kittel propose l'interprétation suivante : Soit un grain sphérique de diamètre $d$, d'aimantation $+J_{\mathrm{s}}$, à l'intérieur duquel sous l'influence du champ antagoniste $-H_{\mathrm{c}}$ s'est formée une paroi de Bloch d'épaisseur $\delta$, assimilable comme le montre la figure 1 , à une calotte sphérique d'épaisseur $\delta$ et de diamètre à la base égal à $p$. Pour que cette paroi prenne naissance et se propage, il faut que l'énergie de formation de la paroi, égale approximativement à $\pi p^{2} \gamma / 4$, où $\gamma$ est égal à l'énergie superficielle de paroi, soit égale à la somme de l'énergie dans le champ extérieur $H_{\mathrm{c}}$ et de l'énergie magnétostatique gagnées à la suite du retournement de l'aimantation à l'intérieur de la calotte. Cette condition s'écrit :

$$
\frac{1}{4} \pi p^{2} \gamma=\frac{1}{8} \pi p^{2} \delta H_{\mathrm{c}} J_{\mathrm{s}}+\frac{1}{2} V J_{\mathrm{s}}^{2} \frac{\delta}{2 d},
$$


où $V$ est le volume de la sphère. En tenant compte de la relation géométrique $p^{2}=4 \delta d_{\imath}$ cette relation s'écrit :

$$
H_{c} / H_{0}=1-\left(d / d_{0}\right)
$$

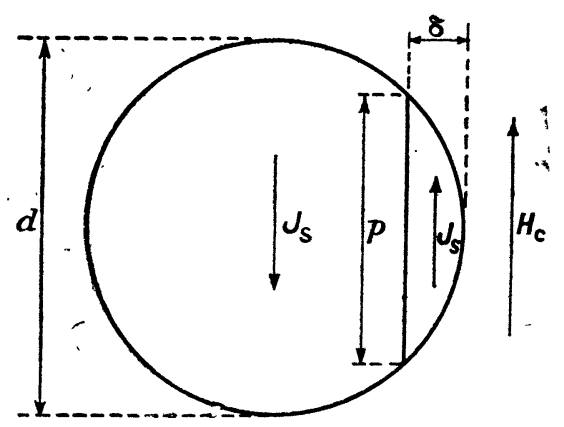

FIg. 1. - Phase initiale de la formation d'un noyau de renversement dans uns sphère (d'après Kittel).

après avoir posé $H_{0}=2 \gamma / \delta J_{\mathrm{s}}$ et $d_{0}=24 \gamma / J_{\mathrm{B}}^{2}$, et montre que le champ coercitif $H_{\mathrm{c}}$ croît à mesure que le diamètre $d$ du grain diminue.

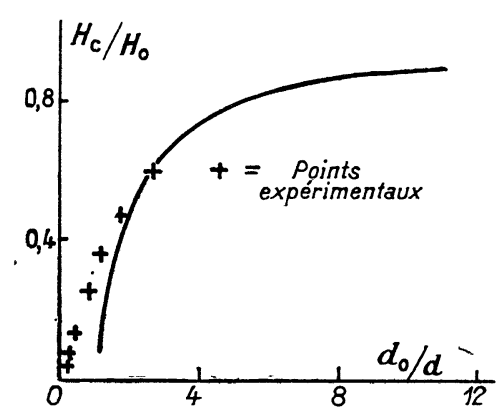

Fig. 2. - Comparaison de la formule théorique de Kitte avec les résultats expérimentaux de Guillaud sur Mn-Bi.

La figure 2, empruntée à Kittel, permet de comparer la formule théorique 2) aux résultats expérimentaux de Guillaud [2], après avoir admis $H_{0}=20000$ et $d_{0}=9.10^{-3} \mathrm{~cm}$ tandis que le calcul théorique donne $H_{0}=40000$ et

$$
d_{0}=7 \cdot 10^{-3} \mathrm{~cm} \text {. }
$$

L'accord est passable.

Il convient néanmoins de faire certaines réserves sur la validité de l'équation 1) et en particulier sur l'expression de la diminution de l'énergie magnétostatique donnée par le deuxième terme du second membre : l'auteur l'obtient par des considérations d'analyse dimensionnelle et considére cette énergie comme proportionnelle à $\delta / 2 d$. Selon ce raisonnement, elle pourrait tout aussi bien être proportionnelle à $(\delta / 2 d)^{n}, n$ étant quelconque : en fait, comme nous allons le montrer, $n$ est égal à 2 .

Nous avons en effet autrefois calculé que l'énergie magnétostatique $W_{m}$ d'un tel système, pour une valeur quelconque de $\delta, s^{\prime}$ écrirait [4]:

$$
W_{m}=\frac{1}{2} \pi^{2} J_{\beta}^{2} d^{3} \sum_{-1}^{\infty} \frac{n(n+1)}{(2 n+1)^{2}} c_{n}^{2},
$$

avec la notation :

$$
c_{n}=\frac{P_{n-2}-P_{n}}{2 n-1}-\frac{P_{n}-P_{n+2}}{2 n+1},
$$

où $P_{n}(x)$ est le polynome de Legendre d'indice $n$ et où $x=1-(2 \delta / d)$. Quand $x$ est voisin de l'unité, $W_{m}$ s'écrit :

$$
W_{m}-\frac{2}{3} \pi V J_{\frac{2}{8}}^{2}\left[1-3\left(\frac{2 \delta}{d}\right)^{2}+\mathrm{o}\left(\frac{2 \delta}{d}\right)^{4}+\ldots\right]
$$

Il est facile de vérifier que la diminution de de l'énergie magnétostatique due à l'apparition d'une calotte sphérique d'épaisseur $\delta$, petite vis-àvis de $d$, aimantée en sens inverse du reste de la sphère, est simplement égale à la variation d'énergie provoquée par le retournement de l'aimantation $J_{s}$, dans un volume égal à celui de la calotte sphérique et dans un champ magnétique égal au champ démagnétisant $H_{d}=\frac{4}{3} \pi J$, de la sphère. Ce résultat très simple aurait pu être écrit $a$ priori.

Dans ces conditions, l'équation 1) doit s'écrire :

$$
\frac{1}{4} \pi p^{2} \gamma=\frac{1}{4} \pi p^{2} \delta\left(H_{c}+\frac{4}{3} \pi J_{s}\right) J_{s}
$$

ou

$$
H_{c}=\frac{\gamma}{\delta J_{s}}-H_{d} .
$$

Cette formule montre, contrairement à l'expérience, que le champ coercitif ne dépend pas des dimensions du grain. La théorie de Kittel est donc insuffisante. L'accord avec l'expérience que traduit la figure 2 est donc purement fortuit.

3. Champ de nucléation et champ de propagation. - A l'image des conceptions qui ont été développées à propos des substances massives, il paraît nécessaire d'introduire dans l'étude des grains multidomaines deux champs critiques : un champ de départ, ou mieux champ de nucléation, égal au champ magnétique nécessaire pour créer un noyau de renversement d'aimantation, et un champ de propagation correspondant au travail à dépenser pour retourner l'aimantation de l'ensemble du grain. Le champ coercitif est égal au plus grand de ces deux champs critiques.

Dans les substances massives, telles que le fer, le nickel et leurs alliages, le champ de nucléation est généralement plus petit que le champ de propagation de sorte que le champ coercitif est simplement égal à ce dernier. Les théories du champ de propagation sont classiques et fournissent des résultats très acceptables. Dans certains cas excep- 
tionnels, comme celui des ferro-nickels étirés et tendus étudiés par Sixtus et Tonks [5], le champ de nucléation devient plus grand que le champ de propagation. Aucune théorie satisfaisante n'en a été donnée : on connaît seulement les conditions suivant lesquelles grandit un noyau de renversement déjà formé mais on ignore complètement le mode de formation de tels noyaux.

Dans le cas des grains, on ignore également le mode de formation des noyaux de renversement et il n'est rien possible de dire sur la théorie des relations qui lient les dimensions du grain au champ de nucléation. Quant à la théorie du champ de propagation, elle prend dans ce cas un aspect particulier que nous nous proposons d'examiner maintenant.

4. Le champ de propagation des grains multidomaines. - Au cours de la description d'un cycle d'hystérésis, un grain passe deux fois par un état de moment magnétique total nul. Un tel état de moment nul correspond à une certaine subdivision en domaines élémentaires d'énergie totale $W_{T}$, dont une partie correspond à l'énergie de parois et l'autre à l'énergie magnétocristalline des domaines de fermeture dont l'aimantation spontanée n'est pas dirigée suivant une direction de facile aimantation. Si l'on admet que la formation et la destruction d'une telle structure est un processus irréversible, l'énergie dépensée au cours de la description d'un cycle d'hystérésis, qui est de l'ordre de $2 H_{\mathrm{c}} J_{\mathrm{s}}$, doit être utilisée à créer deux fois de suite cette structure et on obtient l'égalité approximative :

$$
V H_{\mathrm{c}} J_{\mathrm{s}} \sim W_{T},
$$

où $V$ est le volume du grain.

Pour avoir une idée de l'ordre de grandeur de $\mathrm{W}_{T}$, nous supposons avoir affaire à des grains cubiques d'arête $D$, d'une substance uniaxe dont l'axe est direction de facile aimantation. Lorsque

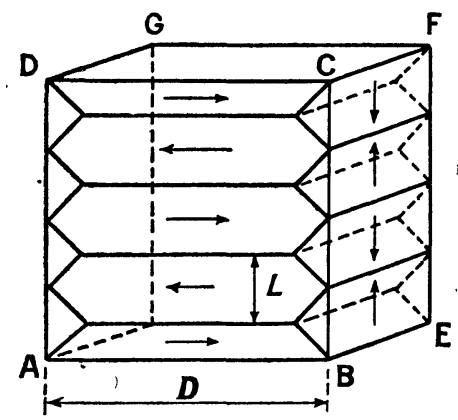

Frg. 3. - Subdivision d'un grain cubique en domaines élémentaires, avec un moment magnétique total nul.

son moment magnétique total est nul, le grain est subdivisé, comme le montre la figure 3 , en domaines lamellaires d'épaisseur $L$ aimantés parallèlement à l'axe, fermés sur la face BCFE et la face opposée par des domaines prismatiques aimantés norma- lement à l'axe. Du point de vue classique, l'énergie libre du cube eśt donnée par l'expression :

$$
W_{T}=\gamma D^{3} L^{-1}+\frac{1}{2} K D^{2} L
$$

dans laquelle le premier terme du second membre représente l'énergie de paroi et le second terme l'énergie magnétocristalline des domaines prismatiques de fermeture.

5. Énergie magnétostatique des parois de petites dimensions. - En réalité, cette expression n'est valable que pour des grains dont l'arête $D$ est très grande vis-à-vis de l'épaisseur de paroi. Il faut, en effet, tenir compte de ce que l'aimantation spontanée tourne à l'intérieur de l'épaisseur de paroi et fait apparaître des pôles magnétiques sur la face $A B C D$ du cube et sur la face opposée. Ces pôles sont répartis avec une densité $+J_{\mathrm{s}}$ et $-J_{\mathrm{s}}$ sur des bandes distantes de L dont la largeur $a$ est de l'ordre de la moitié de l'épaisseur de paroi. L'énergie libre est ainsi augmentée : elle est minimale lorsque les bandes sont alternativement positives et négatives.

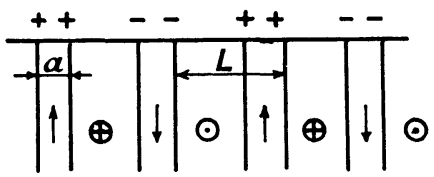

FIG. 4. - Bandes distantes de L et de largeur a portant alternativement des charges positives et des charges négatives.

Considérons une série de bandes parallèles, de largeur $a$, réparties sur un plan, à la distance $L$ les unes des autres ( $f i g .4$ ) et portant alternativement des densités magnétiques égales à $+J_{\mathrm{s}}$ et - $J_{\mathrm{s}}$; un calcul simple montre que l'énergie magnétostatique correspondante est donnée, par $\mathrm{cm}^{2}$ de plan, par l'expression :

$$
\mathcal{E}=\frac{8 L J_{\mathrm{s}}^{2}}{\pi^{2}} \sum_{n=0}^{\infty} \frac{1}{(2 n+1)^{3}} \sin ^{2} \frac{(2 n+1) a \pi}{2 L} .
$$

Cette expression se réduit, pour des bandes contiguës $(L=a)$, ̀̀ :

$$
\mathcal{E}=0,8525 L J_{\mathbb{8}}^{2}
$$

et pour des bandes étroites $(a \ll L)$ à :

$$
\mathcal{E}=\frac{a^{2} J_{\mathrm{s}}^{2}}{L}\left[\frac{3}{2}+\log \frac{2 L}{a \pi}-\frac{a^{2} \pi^{2}}{72 L^{2}}+0 a^{4}+\ldots\right] .
$$

Cette énergie n'est pas négligeable. Considérons par exemple dans du fer

$$
\left(J_{\mathrm{s}}=1700 ; a=10^{-5} \mathrm{~cm}\right)
$$

un système de parois situées à $5 \mu$ les unes des autres ; la formule 6 donne, pour une paroi et une longueur de $1 \mathrm{~cm}$, une énergie de $1,5.10^{-3} \mathrm{erg}$. 
Comme l'énergie superficielle d'une paroi de $180^{\circ}$ dans le fer est voisine de $1,4 \mathrm{erg} / \mathrm{cm}^{2}$, ce résultat indique que des parois de $10 \mu$ de largeur possèdent une énergie qui est en réalité deux fois plus grande que la valeur classique donnée par la formule de Bloch.

Dans la théorie classique de Bloch, l'épaisseur et l'énergie d'une paroi résultent d'un compromis entre l'énergie d'échange et l'énergie magnétocristalline, mais le calcul n'est valable en toute rigueur que pour des parois dont la largeur est grande (une centaine de fois au moins) devant l'épaisseur. Quand ce n'est pas le cas, lorsqu'il s'agit par exemple des parois qui prennent naissance dans un grain ou un cristallite de dimension inférieure à $20 \mu$, l'épaisseur et l'énergie d'une paroi résultent plutôt d'un compromis entre l'énergie d'échange et l'énergie magnétostatique définie par la formule (6).

6. Calcul du champ coercitif d'un petit grain multidomaine. - Appliquons ces considérations au calcul de l'énergie $W_{T}$ du grain eubique envisagé plus haut et soit $a$ une longueur qui soit de l'ordre de la moitié de l'épaisseur, a priori inconnue, de la paroi ; l'énergie totale du cube s'écrit en première approximation :

$W_{T}=\frac{1}{2} K D^{2} L+\frac{1}{4} \gamma_{0}^{2} K^{-1} D^{3} L^{-1} a^{-1}+6,7 J_{\mathrm{s}}^{2} a^{2} D^{2} L^{-1}$

Dans cette expression, le premier terme $d u$ second membre représente l'énergie magnétocristalline des domaines prismatiques de fermeture ; le second terme représente l'énergie d'échange des parois principales exprimées en fonction de $\gamma_{0}$, énergie superficielle d'une paroi infinie calculée par la formule classique de Bloch ; enfin, le dernier terme est l'énergie magnétostatique correspondant à l'expression (6) qui a été simplifiée en remplaçant $L / a$ par 10 dans le logarithme qui figure au second membre, ce qui ne conduit jamais à une grande erreur, compte tenu des grossières approximations faites ailleurs. Nous négligeons à l'intérieur des parois l'énergie magnétocristalline.

Les inconnues $a$ et $L$ se déterminent en écrivant que $W_{T}$ est minimum par rapport à ces deux variables. Le calcul est immédiat et donne :

$$
W_{T}=1,7 \gamma_{0}^{1 / 3} K^{1 / 6} J_{\mathbf{s}}^{1 / 3} D^{7 / 3} \text {. }
$$

Si cette énergie est de l'ordre de grandeur de $D^{3} H_{\mathrm{c}} J_{\mathrm{s}}$, on peut écrire finalement :

$$
H_{\mathrm{c}}-1,7 \gamma^{13} K^{1 / 6} J_{\mathrm{s}}^{-2 / 3} D^{-2 / 3} \text {. }
$$

Le champ coercitif d'un petit grain multidomaine varierait ainsi comme la puissance $2 / 3$ de l'inverse de son diamètre, ce qui cadre assez bien avec les résultats expérimentaux.

Avec $\gamma_{0}=1 \mathrm{erg} / \mathrm{cm}^{2}, \quad K=10^{5} \mathrm{erg} / \mathrm{cm}^{3}$, $J_{\mathrm{s}}=450 \mathrm{cgs}$, valeurs qui conviennent pour la magnétite, on trouve pour des diamètres de $10 \mu$, $1 \mu$ et $0,1 \mu$ des champs coercitifs respectivement égaux à 20,90 et 200 oe. Ces valeurs sont deux à trois fois plus petites que les valeurs expérimentales, peut-être parce que la théorie a négligé l'énergie de formation des noyaux de renversement.

Dans le manganèse-bismuth, la nucléation doit être beaucoup plus difficile que dans la magnétite car l'énergie de champ démagnétisant est, relativement à l'énergie d'anisotropie magnétocristalline, beaucoup plus faible que dans la magnétite ; corrélativement, on constate que le champ coercitif calculé suivant des procédés analogues à ceux qui ont été exposés plus haut est dix fois plus petit que les valeurs expérimentales de Guillaud. Au contraire, pour le fer, la théorie précédente doit s'appliquer mieux que dans la magnétite car l'aimantation spontanée est très grande : l'énergie de nucléation est empruntée simplement à l'énergie de champ démagnétisant.

7. Les couches très minces. - Les couches très minces qui nous intéressent ici sont celles dont l'épaisseur est assez faible pour que le champ démagnétisant amène en chaque point l'aimantation spontanée à être parallèle au plan de la couche : d'après un calcul de Kittel [6], il faut pour cela que l'épaisseur soit inférieure à $3 \cdot 10^{-5} \mathrm{~cm}$. En réalité, cette épaisseur critique doit être deux à trois fois plus grande si l'on tient compte du fait que l'énergie superficielle des petites parois qui peuvent éventuellement se former possède, comme nous l'avons déjà fait remarquer plus haut, une valeur plus grande que la valeur classique relative aux grandes parois.

Dans ces couches très minces, l'aimantation spontanée, en l'absence de perturbations extérieures, s'oriente dans le plan de la couehe suivant la direction d'énergie minimale : les deux sens sont possibles et correspondent à deux catégories de domaines élémentaires séparés par des parois à $180^{\circ}$, perpendiculaires au plan de la couche et parallèles à la direction de facile aimantation. Ainsi, même dans les couches très minces, subsiste le mécanisme de variation de l'aimantation par déplacement de paroi : ces couches devraient ainsi présenter un champ-coercitif faible, comparable à celui des substances massives.

8. - Champ coercitif de propagation de paroi dans les couches très minces. - En réalité, il existe dans les couches três minces des causes de perturbations qui leur sont particulières et qui contribuent à élever le champ coercitif : il s'agit en particulier de l'épaisseur de la couche qui n'est certainement pas uniforme et qui doit présenter, à l'échelle de quelques dizaines d'angströms, un aspect chaotique.

Supposons donc que la surface d'une couche 
mince d'épaisseur moyenne $D$ présente l'aspect ondulé de la figure 5 et soit une paroi $P$, d'abscisse

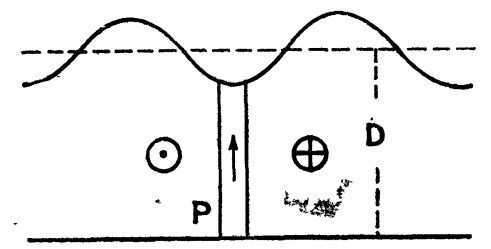

FIG. 5.

$\mathrm{x}$, séparant deux domaines aimantés antiparallèlement normalement. L'énergie de paroi $E_{\mathbf{p}}$ est une fonction de $x$ :

$$
E_{\mathrm{P}}=\left(D+h \sin \frac{x}{h}\right) \gamma
$$

dans laquelle pour simplifier nous supposons que l'amplitude d'une perturbation de période $2 \pi h$ est égale à $h$.

La paroi, d'abord située dans un champ nul en un minimum de $E_{\mathrm{P}}$, subit sous l'action d'un champ $H$ croissant normal au plan du tableau une pression sensiblement égale à $2 D H J_{\mathrm{s}}$, si $h / D$ est petit : elle se déplace d'abord réversiblement puis subit un grand déplacement irréversible au moment où le champ atteint une valeur critique donnée par:

$$
H_{\mathrm{c}}=\frac{\gamma}{2 J_{\mathrm{s}} D}
$$

$h$ a disparu de cette formule : le champ coercitif est inversement proportionnel à l'épaisseur de la couche, dans la mesure oû $\gamma$ est constant.

Cette formule n'est valable que si l'épaisseur de la paroi est petite vis-à-vis de $h$ : pour être autorisé à l'appliquer, il faut donc qu'il existe dans la couche mince des fluctuations d'épaisseur au moins aussi grandes que l'épaisseur de paroi (100 $\AA$ pour les couches de fer de $1000 \AA$ d'épaisseur).

La constante $\gamma$ qui figure dans l'équation 9 n'est pas égale à l'énergie superficielle $\gamma_{0}$ d'une paroi infinie. Il faut tenir compte des pôles magnétiques créés à l'intérieur de la paroi par la composante de l'aimantation spontanée. En utilisant la méthode qui a été décrite plus haut, on trouve que $\gamma$ est lié à $\gamma_{0}$ par la formule suivante, obtenue en remplaçant dans un esprit de simplification $L / a$ par 10 dans le logarithme de l'équation 6 :

$$
\left(\frac{\gamma}{\gamma_{0}}\right)^{3}=2,9 \frac{\gamma_{0} J_{\mathrm{s}}^{2}}{K^{2} D}
$$

Avec $\gamma_{0}=1,4 \mathrm{erg} / \mathrm{cm}^{2} ; \quad J_{\mathrm{s}}=1700 \mathrm{cgs}$; $K=10^{5} \mathrm{ergs} / \mathrm{cm}^{3} ;$ on trouve que $\gamma$ est égal à $\gamma_{0}$ pour $D=12 \mu$ environ. La formule n'est donc valable qu'au-dessous de cette limite. Pour une épaisseur $D$ de $10^{-5} \mathrm{~cm}$, on trouve dans le cas du fer que l'énergie de paroi est voisine de $7 \mathrm{erg} / \mathrm{cm}^{2}$, c'est-à-dire 5 fois plus grande que l'énergie limite.
Corrélativement, on trouve que $a$, qui est de l'ordre de grandeur de la moitié de l'épaisseur totale de paroi, est une fonction de $D$ donnée par l'expression :

$$
a^{3}=0,0187 \frac{\gamma_{0}^{2} D}{K J_{\mathrm{B}}^{2}} .
$$

Pour $D=10^{-5} \mathrm{~cm}$, on trouve $a=10^{-6} \mathrm{~cm}$; c'est une épaisseur 10 fois plus faible que celle des parois normales de largeur infinie.

En remplaçant $\gamma$ dans la formule 9 par sa valeur tirée de la relation 10 on obtient finalement l'expression :

$$
H_{\mathrm{c}}=0.71\left(\frac{\gamma_{0}^{4}}{K^{2} J_{\mathrm{g}}}\right)^{\frac{1}{3}} \frac{1}{D^{4 / 3}},
$$

qui montre que le champ coercitif d'une couche très mince, de surface chaotique, varie comme la puissance 4 /3 de l'inverse de l'épaisseur $D$. L'application numérique, d'après les données citées plus haut, donne un champ coercitif d'environ 200 oe pour des couches de $10^{-5} \mathrm{~cm}$ et de 10 oe pour des couches de $10^{-5} \mathrm{~cm}$ d'épaisseur : c'est donc pour des valeurs de l'épaisseur $D$ un peu supérieures à $10^{-5} \mathrm{~cm}$ qu'il faut s'attendre à une augmentation très notable du champ coercitif conformément aux résultats expérimentaux. La formule 12 donne d'ailleurs des champs coercitifs environ deux fois plus grands que les valeurs expérimentales : il n'y a pas à s'en étonner, étant données les approximations de la théorie et le fait que les accidents sont peut-être plus petits que l'épaisseur de paroi.

Les champs coercitifs très élevés que donne la formule 12 lorsque l'épaisseur est inférieure à $10^{-5} \mathrm{~cm}$ ne sont pas observables, car dans cette région l'aimantation résultante ne change plus de signe par déplacement de paroi, mais plutôt par rotation de l'aimantation spontanée dans le plan de la couche, à l'intérieur des domaines élémentaires : ce champ coercitif de rotation ne doit pas dépasser 150 à 200 oe dans le cas du fer.

\section{DISCUSSION}

M. Van Itterbeek. - Peut-on faire une estimation sur la manière dont la valeur de $H_{0}$ varie en fonction de la température absolue pour le cas des lames épaisses ?

Je voudrais faire encore la remarque suivante : on sait que l'étude des propriétés ferromagnétiques au moyen de la variation de la résistance électrique en fonction du champ, est compliquée. Cependant au point de vue expérimental on est frappé par le fait suivant. On observe qu'à partir d'une certaine épaisseur la variation de la résistance électrique change de signe.

Après mon exposé d'hier, M. Néel a demandé avec quelle fréquience le cycle-d'hystérésis avait 
été décrit. - Je voudrais demander à $M$. Néel si sa question est en rapport avec le phénomène de viscosité.
M. Néel. - Il est possible en effet que le trấnage magnétique joue un rôle important dans les propriétés des lames minces ou des grains fins.

\section{BIBLIOGRAPHIE}

[1] NéEL (L.), C. R. Acad. Sc., 1947, 224, $1488 ;$ ibid., 1947, 224, 1550; Ann. Géophys., 1949, 5, .99; Stoner (E. C.) et Wohlfarth (E. P.), Phil. Trans, 1948, 240,599.

[2] Gotrschalk (V.), Physics, 1935, 6, 127 ; Gotrschalk (V.) et Wartman (F.), $U$. S. Bur. Mines Rep. Investig., 1935, 67, 3268; GuILlaud (G.), Thèse, Strasbourg, 1943.

[3] Kittel (C.), Phys. Rev., 1948, 73, 810.

[4] Néex (L.), Cahiers de Phys., 1944, no 25, p. 21.

[5] Sixtus (K. J.) et Tonks (K.), Phys. Rev., 1930, 85, 1441 ; ibid., 1931, 37, $930 ; 1932,42,419 ; 1935,48$, 425.

[6] Kittel (G.), Phys. Re»., 1946, 70, 281 et 965. 